\title{
Obesity in pregnancy
}

\author{
Natalia Sirimi ${ }^{1}$, Dimitrios G. Goulis ${ }^{2}$ \\ ${ }^{1}$ Second Department of Internal Medicine, 251 General Air Force Hospital, Athens, ${ }^{2}$ Unit of Reproductive Endocrinology, \\ First Department of Obstetrics and Gynecology, Aristotle University of Thessaloniki, Greece
}

\begin{abstract}
Obesity, the most common metabolic disorder, concerns, among others, women of reproductive age and, when it occurs before or during pregnancy, constitutes a major risk factor for both maternal and fetal complications. The complications of obesity in a prospective mother include subfertility, miscarriage, thrombo-embolism, hypertensive disorders, metabolic syndrome, preterm delivery and higher frequency of cesarean section. Fetal complications include intrauterine death, congenital anomalies and macrosomia. Moreover, the complications of maternal obesity do not only involve the fetus; they also extend beyond fetal life into childhood and adulthood. The mother's diet during pregnancy creates a metabolic environment that affects fetal growth and may result in later development of metabolic syndrome and cardiovascular disease, a phenomenon known as "developmental origin of adult disease". Since the expectant mother is usually more motivated to accept lifestyle modifications, pregnancy is a period during which obesity can be more effectively managed. The control of body weight during this period is of paramount importance for pregnancy outcome as well as the health status of the mother and the neonate.
\end{abstract}

Keywords: Developmental origin of adult disease, Diet, Metabolic syndrome, Obesity, Physical exercise, Pregnancy

\section{INTRODUCTION}

The alarming increase in the prevalence of obesity worldwide, currently the commonest metabolic disease, has led the World Health Organization (WHO) to consider obesity as one of the most serious global health problems of the 21 st century. ${ }^{1}$ Obesity is defined as excessive body fat to an extent that is highly likely to impair health and increase morbidity and mortal-

Address for correspondence:

Dr. Natalia Sirimi, 40 Messinias Street, 11526 Athens, Greece e-mail: sirnatalia@hotmail.com

Received 23-05-10, Revised 28-07-10, Accepted 15-08-10 ity. Body mass index (BMI) is the most frequently applied indicator of obesity. Thus, individuals with a BMI of 25 to $30 \mathrm{~kg} / \mathrm{m}^{2}$ are categorized as overweight, and those with a BMI over $30 \mathrm{~kg} / \mathrm{m}^{2}$ are considered obese. According to WHO, the prevalence of obesity in pregnancy ranges from 1.8 to $25.3 \% .^{1}$ Obesity before and during pregnancy constitutes a major risk factor for both maternal and fetal complications, ${ }^{2,3}$ while it has been shown that offspring of obese women have a higher likelihood of becoming obese during childhood and of developing metabolic syndrome in adulthood (Figure 1). ${ }^{4}$ Pregnancy is a period during which obesity can be relatively effectively confronted 


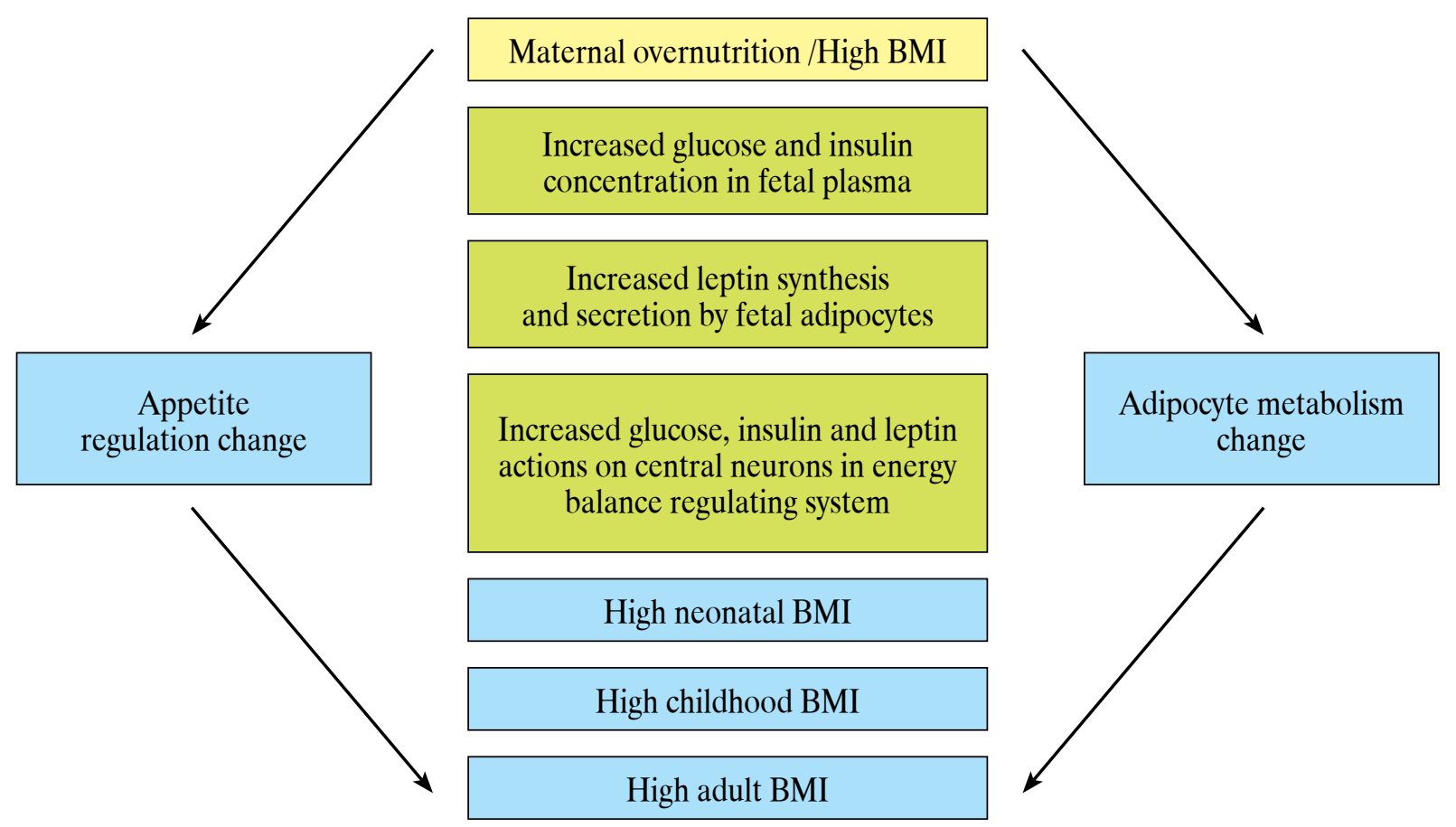

Figure 1. Possible relationships between maternal diet and fetal macrosomia. (From McMillen IC, et al, 2006 ${ }^{4}$ with permission).

as the mother is more disposed to accept lifestyle modifications, such as balanced diet and physical exercise, which will most likely improve fetal health. ${ }^{2}$ The control of body weight during this period is of paramount importance for delivery outcome as well as maternal and neonatal health. In the present communication, data on the effects of obesity prior to and during pregnancy on the health status of mother and fetus will be reviewed.

\section{OBESITY BEFORE PREGNANCY}

Obese, as well as underweight, women have reduced fertility as compared to women with normal body weight, this being observed in both natural conception $^{5}$ and conception achieved by assisted reproduction techniques (ARTs). ${ }^{6}$ Subfertility observed in obese women is partly attributed to the decrease in frequency or complete absence of ovulation. ${ }^{2,3}$ The prevailing cause of oligo- or anovulation is polycystic ovary syndrome (PCOS), which is characterised by central visceral obesity in $40 \%$ of the cases. ${ }^{7}$ Moreover, in a wide context of metabolic disorders, low concentration of serum high-density lipoprotein (HDL) cholesterol, high concentration of triglycerides and low-density lipoprotein (LDL) cholesterol ${ }^{8,9}$ as well as increased concentrations of plasminogen activator inhibitor-1 (PAI-1) tend to co-exist. ${ }^{10,11}$ All these metabolic derangements increase the risk of developing arterial hypertension, coronary heart disease and thrombosis. In addition, hyperinsulinemia promotes the production of testosterone and $\Delta_{4}$-androstendione from the theca cells in the ovary and results in a condition of hyperandrogenemic anovulation, with a phenotype characterized by hirsutism, obesity, acne, seborrhoea and hair loss. ${ }^{12}$

Obesity has been observed to exert a negative impact on ARTs. ${ }^{13}$ Specifically, obesity may adversely affect the duration of ovarian stimulation, number and quality of oocytes and embryos, fertilization rate, embryo transfer rate as well as implantation and pregnancy rates. In obese women, a longer duration of ovarian stimulation and higher doses of gonadotropins are required. Additionally, higher cancellation rates and higher incidence of follicular asynchrony are often observed. ${ }^{13,14}$ The lower number of oocytes retrieved in overweight and obese as compared to normal weight women may be due to the lower number of follicles, technical difficulties or both. Moreover, obesity can prevent proper trans-abdominal ultrasono- 
graphic imaging during embryo transfer. All these mechanisms may be responsible for the decrease in implantation and pregnancy rates in obese women undergoing ART. ${ }^{15}$

\section{OBESITY DURING PREGNANCY}

\section{A. Maternal complications}

The recommended gestational weight gain is 11.5 to $16.0 \mathrm{~kg},(0.5$ to $2.0 \mathrm{~kg}$ for the first trimester and 0.35 to $0.50 \mathrm{~kg}$ per week for the second and third trimesters) (Table 1). ${ }^{16}$ It is strictly recommended for overweight and obese pregnant women to limit weight gain to a minimum and this aim is achieved by a balanced diet of high nutritional value, which results in both weight control and normal embryo growth. Women who are overweight before pregnancy are far more likely to exceed the weight gain recommendations during pregnancy and to experience greater risk of adverse complications, ${ }^{17}$ as outlined below.

\section{Miscarriage}

Although excess body weight has been correlated with increased risk for first-trimester miscarriage, the results of various studies are controversial and far from being conclusive..$^{18-20}$

\section{Thrombo-embolism}

Pregnancy per se constitutes a prothrombotic state characterized by an increase in the plasma concentration of coagulation factors I, VII, VIII and X, a decrease in protein $\mathrm{S}$ and inhibition of fibrinolysis. ${ }^{21}$ These changes in combination with other risk factors, such as advanced maternal age, high parity, cesarean section, pre-eclampsia and obesity, result in an increased risk for venous thrombosis. According to several studies, obesity (BMI $>30 \mathrm{~kg} / \mathrm{m}^{2}$ ) doubles the risk of thrombosis by increasing the concentration of factors VIII and IX, but not of fibrinogen. ${ }^{22}$ Furthermore, BMI $>25 \mathrm{~kg} / \mathrm{m}^{2}$ combined with oral contraceptive pills greatly increases ${ }^{22}$ the risk of thrombosis among women aged $15-45$ years.

\section{Gestational diabetes}

Approximately $3-10 \%$ of women will be affected by gestational diabetes. ${ }^{3}$ Although many factors contribute to this, such as ethnic origin, age and family history, obesity constitutes an independent risk factor as the incidence of gestational diabetes is two- to three-fold higher in obese and overweight as compared to normal weight women. ${ }^{3}$ Moreover, obesity and diabetes play independent roles in determining fetal size. Women with gestational diabetes and normal body weight who control glycemia with diet, insulin or anti-diabetic drugs present an incidence of neonatal macrosomia comparable to that of women without diabetes. ${ }^{23}$ In addition, insulin treatment prevented macrosomia in overweight and obese women. On the other hand, in obese women on diet who managed to control glycemia, as well as in overweight and obese women who failed to do so, irrespective of treatment, the incidence of macrosomia remained high. ${ }^{23}$

\section{Hypertensive disorders and metabolic syndrome}

Obesity is associated with insulin resistance. Insulin resistance and consequent hyperinsulinemia can result in such conditions as hypertension, hyperlipidemia, degenerative heart disease, hyperuricemia and glucose intolerance or diabetes type 2. Hyperleptinemia, increased PAI-1 concentrations, micro- or macro-proteinuria and endothelium dysfunction have also been reported. This group of disorders has been collectively described as "metabolic syndrome".

Table 1. Recommendations for total and rate of weight gain during pregnancy, according to pre-pregnancy body mass index. (From the Institute of Medicine and National Research Council, 2009). Re-examining the guidelines. Washington, DC: The National Academic Press. ${ }^{16}$

\begin{tabular}{lccc}
\hline & Total weight gain $(\mathbf{k g})$ & & Rates of weight gain at second and third trimester $(\mathbf{k g} /$ week) \\
\cline { 2 - 2 } Pre-pregnancy BMI & Range & $12.5-18.0$ & Mean (range) \\
\hline Underweight: $<18.5 \mathrm{~kg} / \mathrm{m}^{2}$ & $11.5-16.0$ & & $0.51(0.44-0.58)$ \\
Normal weight: $18.5-24.9 \mathrm{~kg} / \mathrm{m}^{2}$ & $7.0-11.5$ & $0.42(0.35-0.50)$ \\
Overweight: $25.0-29.9 \mathrm{~kg} / \mathrm{m}^{2}$ & $5.0-9.0$ & & $0.28(0.23-0.33)$ \\
Obese: $\geq 30.0 \mathrm{~kg} / \mathrm{m}^{2}$ & & $0.22(0.17-0.27)$ \\
\hline
\end{tabular}

Calculations assume a $0.5-2 \mathrm{~kg}$ weight gain in the first trimester. 
During pregnancy, obese women face increased risk of developing hypertension, pre-eclampsia and gestational diabetes. Specifically, women with a BMI $>30 \mathrm{~kg} / \mathrm{m}^{2}$ have a two- to three-fold higher risk for developing pre-eclampsia, while this risk doubles for an increase in BMI prior to pregnancy by $5-7 \mathrm{~kg} / \mathrm{m}^{2}{ }^{24}$ Waist circumference is considered as the most sensitive index of visceral obesity, which is directly associated with an increased risk for hypertensive disorders. At the same time, waist circumference can identify up to $46 \%$ of the subjects who are liable to develop metabolic syndrome within the next five years. ${ }^{25} \mathrm{On}$ the other hand, obesity and a previous pregnancy complicated by pre-eclampsia constitute the main risk factors for developing severe pre-eclampsia in the current pregnancy. Pre-eclampsia is also associated with an increased risk for coronary heart disease in later life. ${ }^{26}$

\section{Preterm delivery}

Current evidence indicates that obesity during pregnancy leads to induced preterm delivery but not spontaneous preterm birth, which is usually encountered in women with low BMI. ${ }^{27,28}$ Nevertheless, the data are still inconclusive. ${ }^{29,30}$

\section{Cesarean section}

Studies suggest a two-fold ${ }^{3}$ increase in the risk for cesarean section in obese women even without additional risk factors. Cesarean section in this group is of great concern, as women who are overweight or obese are more susceptible to post-operative complications, such as excessive blood loss, deep venous thrombosis, wound infection and postpartum uterine infection.

Moreover, obesity has been associated with intra-partum complications and vaginal operative delivery. ${ }^{31,32}$

\section{Long-term complication}

Obese women tend to retain gestational weight gain more frequently than women of normal body weight. ${ }^{17,33}$ It is also very important to note that women who developed gestational diabetes have a 50\% likelihood of developing type 2 diabetes within the following ten years. ${ }^{34}$

\section{B. Fetal complications}

Maternal obesity is associated with an increased risk for perinatal mortality and occurrence of genetic disorders. ${ }^{3}$ The most common complications for the fetus are intrauterine death, genetic disorders and macrosomia. In the long term, large for gestational age (LGA) neonates of obese or diabetic mothers are prone to development of childhood obesity and metabolic syndrome in their adult life. ${ }^{35}$

\section{Fetal death}

Fetal death constitutes a dramatic development of any pregnancy, especially when it takes place in late pregnancy. In some studies, an up to five-fold increase in intrauterine death as well as increased infant mortality rate has been recorded in obese women. ${ }^{36}$ There also appears to be a correlation between maternal BMI and infant mortality rate. ${ }^{37}$

\section{Congenital anomalies}

Prenatal screening for congenital anomalies becomes problematic in obese women due to difficulty of interpreting blood serum indices as well as inability to display the fetal anatomy on the ultrasonogram. ${ }^{3}$ These difficulties could, at least in part, explain the increased incidence of congenital anomalies in fetuses of obese women. Nevertheless, there are data supporting an actual association between maternal obesity and genetic disorders. Specifically, the fetuses of obese mothers have a higher risk of developing abnormalities of the neural tube, as for example spinal bifida, cardiovascular abnormalities, as well as abnormalities of the abdominal wall such as omphalocele. These abnormalities are more prevalent in offspring of women with type 2 diabetes and folic acid deficiency, disorders that often coexist with obesity. Finally, maternal obesity results in an increased admission rate of the offspring to the neonatal intensive care unit. ${ }^{3,38}$

\section{Macrosomia}

The association between maternal obesity and fetal macrosomia has been established by many studies. ${ }^{3}$ Maternal weight and insulin resistance before pregnancy affect fetal growth, as is reflected in the birth weight. ${ }^{39}$ Obesity and insulin resistance alter placental function which, during the last weeks of pregnancy, increases the availability of glucose, free fatty acids and aminoacids to the fetus..$^{40}$ Thus, maternal hyperglycemia induces fetal hyperglycemia 
and, as a consequence, hypertrophy/hyperplasia of the fetal pancreas and hyperinsulinemia. Insulin has a direct effect on cell division that leads to macrosomia. Therefore, women with diabetes are at high risk of delivering macrosomic babies. Obese women even with normal glucose tolerance have a two-fold higher risk of giving birth to macrosomic babies since both conditions are independently correlated to macrosomia. Given that the incidence of obesity is approximately ten-fold that of gestational diabetes, it is evident that maternal lifestyle exerts a great influence on the incidence of fetal macrosomia. Once again, there seems to be a quantitative relationship between maternal BMI and the risk of delivering a macrosomic/LGA neonate. ${ }^{41,42}$ Macrosomia, as well as maternal height and weight, gestational age and number of prior deliveries, are considered reliable predictors of the risk of obstetrical events, such as shoulder dystocia and injury of the branchial plexus. ${ }^{3}$

\section{Long-term complications}

There is considerable evidence that the conplications of maternal obesity extend beyond intrauterine and neonatal life into childhood and adulthood, thus leading to serious life long health problems. ${ }^{43,44}$ Maternal obesity constitutes a risk for childhood obesity, ${ }^{45}$ irrespective of birth weight and of smoking during pregnancy, as well as a risk for the appearance of the metabolic syndrome and cardiovascular disease in adulthood.

There is a large number of pathophysiologic mechanisms that could explain the fact that obese mothers give birth to obese children. Genetic factors are undoubtedly responsible to a certain degree for the tendency of both mother and child to be overweight. Additionally, the impact of the environment is to be taken into account since mother and child typically share the same eating habits. ${ }^{4}$ Moreover, the increase in food intake during pregnancy has a quantitative effect on the fetus, promoting the development of adipose tissue..$^{43}$

Childhood and adolescent obesity exerts a longlasting physical and psychological impact and increases population morbidity and mortality. The increasing prevalence of maternal obesity before and during pregnancy results in a vicious circle of obesity in subsequent generations. Thus, obese mothers give birth to obese daughters, who have a higher risk of suffering from obesity and diabetes during their own pregnancies. ${ }^{4,40}$

\section{Developmental origin of adult disease}

The abnormal development of the fetus results in increased morbidity during childhood, adolescence and adulthood, a phenomenon known as "fetal programming" or "developmental origin of adult disease". ${ }^{46}$ The fetal adjustment to the uterine environment leads to permanent changes in the phenotype (i.e. physical structure, physiology and metabolism) which might not be fully functional in extra-uterine conditions. In order to explain this phenomenon, particularly the connection between fetal development and type 2 diabetes, the hypothesis of the "thrifty phenotype" was formulated. According to this, poor nutrition during intrauterine life, as reflected in low birth weight, results in adverse physiological or morphological characteristics ("developmental plasticity") in certain organs (e.g. pancreas), while it respects others (e.g. brain). When the individual faces environmental changes in postnatal life, such as an increase in calorie intake, these adaptive changes are no longer favorable and lead to disease.$^{35}$ In addition to type 2 diabetes, the "thrifty phenotype" hypothesis possibly accounts for such diseases as hypertension, hyperlipidemia, atherosclerosis, cardiovascular disease and stroke, their common denominator being insulin resistance. ${ }^{47}$

\section{MANAGEMENT}

\section{Before pregnancy}

Obtaining normal weight before pregnancy is an ideal goal since it is of paramount importance not only for conception but also for the outcome of pregnancy. ${ }^{16}$ However, many pregnancies are not planned and, even when they are, very few women consult a specialist on how to lose weight, the main reason being that the majority of women are reluctant to postpone their family planning in order to achieve the target weight. Nevertheless, it is now an established fact that weight loss before pregnancy results in a better chance for conception and increases the percentage of live births for obese women with or without PCOS. ${ }^{2}$

Treatment of obesity before pregnancy is a difficult 
task. It is essential, however, that obese women are fully informed about the risks of obesity and the benefits of weight loss. Management includes improved lifestyle by balanced diet and physical exercise. Medical treatment and bariatric surgery can be considered as second-line options. A low-calorie diet with reduced glycemic load and an adequate amount of proteins and mono-unsaturated fats reduces hyperinsulinemia in six months. ${ }^{48}$ Physical exercise, especially aerobic, results in weight loss as well as in the reduction of serum testosterone, ${ }^{48}$ while anti-diabetic drugs, such as glibenclamide and metformin, might prove to be beneficial and have not proven harmful. Finally, bariatric surgery provides more permanent solutions in the event that the above-mentioned measures have failed. ${ }^{2,48}$

\section{During pregnancy}

Given the fact that obesity or excessive weight gain during pregnancy constitute important risk factors for the mother and the fetus and, moreover, that women who gain excessive weight during pregnancy have a three-fold higher risk of becoming overweight in subsequent pregnancy, ${ }^{17}$ balanced diet and physical exercise during, and if possible before pregnancy are strongly recommended. Social trends which support the view that a pregnant woman should "eat for two" are not scientifically proven. On the other hand, it is essential that pregnant women follow a diet of high nutritional value which includes all food groups, whereas consumption of foods with high fat content and high glycemic index should be kept to a minimum. At the same time, provided that there are no medical or obstetric contra-indications, aerobic exercise of medium intensity has marked beneficial effects. ${ }^{2}$ Thus pregnant women are counseled to exercise on a daily basis at a moderate degree (i.e. walking, swimming, aerobics) for 30 or more minutes. ${ }^{49}$ Nevertheless activities that present a high risk of falls or abdominal injury as well as intense exercise must be avoided. Furthermore, given that exercise reduces the risk of developing pre-eclampsia, glucose intolerance and gestational diabetes, overweight and obese women should be encouraged to abandon sedentary lifestyle. Pregnancy is also the ideal time for other changes in lifestyle, such as quitting smoking and giving up other harmful habits. The mother should be encouraged and motivated to consider this effort as both an investment in the maintenance of her own health as well as in that of her baby. ${ }^{50}$

\section{After pregnancy}

The postpartum period is another suitable time for the mother to adopt healthy lifestyle habits that will allow her not only to lose the excessive weight gained during pregnancy but also to achieve ideal body weight in view of a prospective pregnancy. However, the need to care for the newborn baby, the psychological alterations of the puerperal period and previous failed attempts to lose weight often discourage women from making a serious effort. Thus, the support of the whole family and consultation with a dietician are required in order for the woman to combine healthy diet with physical exercise. While nursing is theoretically thought to facilitate postpartum weight loss, the results of relevant studies do not support such conclusions. ${ }^{51,52}$

Healthcare providers have the potential to prevent high postpartum weight retention and future obesity by monitoring weight gain during pregnancy and giving appropriate advice about recommended amounts of gestational weight gain. Although there has been a substantial improvement in the management of maternal obesity over the last three years, more work is needed specifically concerning psychosocial issues and the public health aspects of maternal obesity. ${ }^{53} \mathrm{To}$ meet the needs of obese pregnant women, maternity services should consider close observation not only of the period of pregnancy but of the postnatal period as well through improving communication between hospital and public health services and developing services that will encourage pregnant women to optimally address all health concerns surrounding their obesity.

\section{CONCLUSIONS}

Maternal obesity constitutes a serious health risk for both mother and fetus, the impact of which increases with the degree of obesity. A non-balanced diet during pregnancy contributes not only to abnormal fetal development and subsequently increased neonatal morbidity and mortality but also to increased morbidity during childhood, adolescence and adulthood ("developmental origins of adult disease"). A systematic effort for weight reduction is imperative in 
order to avoid transmitting obesity from generation to generation. Achieving this goal will most likely result in a sharp decrease in fetal and neonatal morbidity and mortality and will improve the outcome of offspring and of future pregnancies.

\section{REFERENCES}

1. Guelinckx I, Devlieger R, Beckers K, Vansant G, 2008 Maternal obesity: pregnancy complications, gestational weight gain and nutrition. Obes Rev 9: 140-150.

2. Birdsall KM, Vyas S, Khazaezadeh N, Oteng-Ntim E, 2009 Maternal obesity: a review of interventions. Clin Pract 63: 494-507.

3. Yogev Y, Catalano PM, 2009 Pregnancy and Obesity. Obstet Gynecol Clin N Am 36: 285-300.

4. McMillen IC, Edwards LJ, Duffield J, Muhlhausler BS, 2006 Regulation of leptin synthesis and secretion before birth: implications for the early programming of adult obesity. Reproduction 131: 415-427.

5. Zaadstra BM, Seidell JC, Van Noord PA, et al, 1993 Fat and female fecundity: prospective study of effect of body fat distribution on conception rates. Br Med J 306: 484-487.

6. Crosignani PG, Ragni G, Parazzini F, et al, 1994 Anthropometric indicators and response to gonadotrophin for ovulation induction. Hum Reprod 9: 420-423.

7. Carmina E, Lobo RA, 1999 Polycystic ovary syndrome (PCOS): arguably the most common endocrinopathy is associated with significant morbidity in women. J Clin Endocrinol Metab 84: 1897-1899.

8. Wild RA, Painter PC, Coulson PB, Carruth KB, Ranney GB, 1985 Lipoprotein lipid concentrations and cardiovascular risk in women with polycystic ovary syndrome. J Clin Endocrinol Metab 61: 946-951.

9. Conway GS, Argawal R, Betteridge DJ, Jacobs HS, 1992 Risk factors for coronary artery disease in lean and obese women with the polycystic ovary syndrome. Clin Endocrinol 37: 119-125.

10. Dahlgreen E, Janson PO, Johansson S, Lapidus L, Lindstedt G, Tengborn L, 1994 Hemostatic and metabolic variables in women with polycystic ovary syndrome. Fertil Streril 61: 455-460.

11. Sampson M, Kong C, Patel A, Unwin R, Jacobs HS, 1996 Ambulatory blood pressure profiles and plasminogen activator inhibitor (PAI-I) activity in lean women with and without the polycystic ovary syndrome. Clin Endocrinol 45: 623-629.

12. Jacobs HS, Conway GS, 1999 Leptin, polycystic ovaries and polycystic ovary syndrome. Hum Reprod Update 5: 166-171.

13. Erel CT, Senturk LM, 2009 The impact of body mass index on assisted reproduction. Curr Opin Obstet Gynecol 21: 228-235.

14. Metwally M, Ledger WL, Li TC, 2008 Reproductive endocrinology and clinical aspects of obesity in women. Ann N Y Acad Sci 1127: 140-146.

15. Maheshwari A, Stofberg L, Bhattacharya S, 2007 Effect of overweight and obesity on assisted reproductive technology: a systematic review. Hum Reprod Update 13: 433-444.

16. IOM (Institute of Medicine) and NRC (National Research Council), 2009 Weight Gain During Pregnancy: Reexamining the Guidelines. Washington, DC: The National Academies Press.

17. Gunderson EP, 2009 Childbearing and obesity in women: Weight before, during, and after pregnancy. Obstet Gynecol Clin N Am 36: 317-332.

18. Wang JX, Davies MJ, Norman RJ, 2002 Obesity increases the risk of spontaneous abortion during infertility treatment. Obes Res 10: 551-554.

19. Lashen H, Fear K, Sturdee DW, 2004 Obesity is associated with increased risk of first trimester and recurrent miscarriage: matched case-control study. Hum Reprod 19: 1644-1646.

20. Weiss JL, Malone FD, Emig D, et al, 2006 FASTER Research Consortium. Obesity, obstetric complications and caesarean delivery rate: a population-based screening study. Am J Obset Gynecol 190: 1091-1097.

21. Greer IA, 1994 Haemostasis and thrombosis in pregnancy. In: Bloom AL Forbes CD, Thomas DP et al (eds). Haemostasis and Thrombosis in Pregnancy, Churchill Livingstone, Edinburgh (UK), pp 987-1015.

22. Abdollahi M, Cushman M, Rosendaal FR, 2003 Obesity: risk of venous thrombosis and the interaction with coagulation factor levels and oral contraceptive use. Thromb Haemost 89: 493-498.

23. Leikin E, Jenkins JH, Graves WL, 1987 Prophylactic insulin in gestational diabetes. Obstet Gynecol 70: 587 592.

24. O’Brien TE, Ray JG, Chan WS, 2003 Maternal body mass index and the risk of pre-eclampsia: a systematic review. Epidemiology 14: 368-374.

25. Sattar N, Clark P, Holmes A, et al, 2001 Antenatal waist circumference and hypertension risk. Obstet Gynecol 97: 268-271.

26. Sattar N, Greer IA, 2002 Pregnancy complications and maternal cardiovascular risk: opportunities for intervention and screening? Br Med J 325: 157-160.

27. Smith GCS, Shah I, Pell JP, et al, 2007 Maternal obesity in early pregnancy and risk of spontaneous and elective preterm deliveries: a retrospective cohort study. Am J Public Health 97: 157-162.

28. Bhattacharya S, Campbell DM, Liston WA, et al, 2007 Effect of body mass index on pregnancy outcomes in nulliparous women delivering singleton babies. BMC Public Health 24: 168.

29. Hendler I, Goldenberg RL, Mercer BM, et al, 2005 The Preterm Prediction Study: association between maternal body mass index and spontaneous and indicated preterm birth. Am J Obstet Gynecol 192: 882-886. 
30. Ramachenderan J, Bradford J, McLean M, 2008 Maternal obesity and pregnancy complications: a review. Aust N Z J Obstet Gynaecol 48: 228-235.

31. Kaiser PS, Kirby RS, 2001 Obesity as a risk factor for cesarean in a low-risk population. Obstet Gynecol 97: 39-43.

32. Yu CK, Teoh TG, Robinson S, 2006 Obesity in pregnancy BJOG 113: 1117-1125.

33. Linne Y, Neovious M, 2006 Identification of women at risk of adverse weight development following pregnancy. Int J Obes 30: 1234-1239.

34. Linne Y, Barkeling B, Rossner S, 2002 Natural course of gestational diabetes mellitus: long term follow up of women in the SPAWN study. BJOG 109: 1127-1131.

35. Heindela JJ, von Saalb FS, 2009 Role of nutrition and environmental endocrine disrupting chemicals during the perinatal period on the aetiology of obesity. Molecular and Cellular Endocrinology 304: 90-96.

36. Nohr EA, Bech BH, Davies MJ, et al, 2005 Prepregnancy obesity and fetal death. Obstet Gynecol 106: 250-259.

37. Salihu HM, Dunlop A, Hedayatzadeh M, et al, 2007 Extreme obesity and risk of stillbirth among black and white gravidas. Obstet Gynecol 110: 552-557.

38. Stothard KJ, Tennant PW, Ruth B, Rankin J, 2009 Maternal overweight and obesity and the risk of congenital anomalies: A systematic review and meta-analysis. JAMA 301: 636-650.

39. Catalano P, Drago N, Amini S, 1995 Maternal carbohydrate metabolism and its relationship to fetal growth and body composition. Am J Obstet Gynecol 172: 1464-1470.

40. Murphy VE, Smith R, Giles WB, Clifton VL, 2006 Endocrine regulation of human fetal growth: The role of the mother, placenta, and fetus. Endocr Rev 27: 141-169.

41. Ehrenberg HM, Mercer BM, Catalano PM, 2004 The influence of obesity and diabetes on the prevalence of macrosomia. Am J Obstet Gynecol 191: 964-968.

42. Beaten JM, Bukusi EA, Lambe M, 2001 Pregnancy complications and outcomes among overweight and obese nulliparous women. Am J Public Health 91: 436-440.
43. Ozanne SE, 2009 The long-term effects of early postnatal diet on adult health. Adv Exp Med Biol 639: 135-144.

44. Symonds ME, Sebert SP, Budge H, 2009 The impact of diet during early life and its contribution to later disease: critical checkpoints in development and their long-term consequences for metabolic health. Proc Nutr Soc 68: 416-421.

45. Kitsantas P, Pawloski LR, Gaffney KF, 2010 Maternal prepregnancy body mass index in relation to Hispanic preschooler overweight/obesity. Eur J Pediatr [Epub ahead of print].

46. Gardner DS, Rhodes J, 2009 Developmental origins of obesity: programming of food intake or physical activity? Adv Exp Med Biol 646: 83-93.

47. Kajantie E, 2008 Early-life events. Effects on aging. Hormones 7: 101-113.

48. The Thessaloniki ESHRE/ASRM-Sponsored PCOS Consensus Workshop Group, 2008 Consensus on infertility treatment related to polycystic ovary syndrome. Fertil Steril 89: 505-522.

49. American College of Obstetricians and Gynecologists (ACOG) committee opinion, 2002 Exercise during pregnancy and the postpartum period. Number 267. Int J Gynaecol Obstet 77: 79-81.

50. Phelan S, 2010 Pregnancy: a "teachable moment" for weight control and obesity prevention. Am J Obstet Gynecol 202: 135.e1-8.

51. Howie GJ, Sloboda DM, Kamal T, et al, 2009 Maternal nutritional history predicts obesity in adult offspring independent of postnatal diet. J Physiol 587(Pt 4): 905915.

52. Armitage JA, Khan IY, Taylor PD, et al, 2004 Developmental programming of the metabolic syndrome by maternal nutritional imbalance: how strong is the evidence from experimental models in mammals? J Physiol 561(Pt 2): 355-377.

53. Heslehurst N, Moore H, Rankin J, Ells LJ, Wilkinson JR, Summberbell CD, 2010 How can maternity services be developed to effectively address maternal obesity? A qualitative study. Midwifery [Epub ahead of print]. 